\title{
MORPHOLOGIC VARIATION IN PNEUMOCOCCUS
}

\section{Control of Pneumococcal Morphology through Transformation REACTIONS*}

BY ROBERT AUSTRIAN, M.D.

(From the Biological Division, Department of Medicine, The Johns Hopkins University School of Medicine, Baltimore)

(Received for publication, March 7, 1953)

In the preceding paper (1), a hitherto undefined morphologic variant of pneumococcus has been described and its relation to the three previously recognized morphologic variants of this species set forth. The data presented revealed, as had earlier studies $(2,3)$, that two of the principle factors influencing morphologic patterns in pneumococcus are the mode of cellular separation after division and the production of type-specific capsular polysaccharide. Both these pneumococcal characters have been shown to be subject to control through transformation reactions $(4,5)$ and in addition, to be inherited independently of one another in such reactions $(6,7)$. If these findings are applicable generally, it should be possible to alter predictably any competent pneumococcal morphologic variant through transformation reactions with the transforming principles of any of the other morphologic variants. The present experiments are concerned with an examination of this problem.

\section{Materials and Methods}

1. Nomenclature.-The nomenclature used to describe the morphologic variants of pneumococcus is that employed in the preceding paper (1).

2. Preparation of Transforming Principles and Techniques of Transformation Reactions.Transforming principles (T.P.) were prepared according to the method of MacLeod and Krauss (8). In all experiments save those concerned with the possible exchange of desoxyribose nucleic acids controlling production of type-specific capsular polysaccharide, the technique for carrying out the transformation reaction was that used by these two authors. $0.1 \mathrm{cc}$. of a $10^{-4}$ dilution of an 18 hour blood broth culture of the strain to be transformed was inoculated into $2 \mathrm{cc}$. of charcoal-absorbed neopeptone broth containing 5 per cent human pleural fluid and $0.1 \mathrm{cc}$. of the appropriate transforming principles. Cultures containing no T.P. were prepared as controls. After 24 hours' incubation at $37^{\circ} \mathrm{C}$., a loopful of each culture was streaked on the surface of a neopeptone agar plate containing 5 per cent defibrinated rabbit's blood. In experiments yielding both autoagglutinable and diffusely growing variants, both supernatant fluid and sediment from the transformed and control cultures were inoculated on the surface

* This work was supported by a grant-in-aid from the National Institutes of Health, Public Health Service, Federal Security Agency. 
of solid media. The plates were incubated for 24 hours and examined then for preliminary identification of the several morphologic variants under a colony microscope at a magnification of $\mathbf{3 0}$ diameters. Individual clones were then isolated and the variants identified further by the use of the Gram stain and of quellung and agglutination reactions. In addition, to test for the presence of the factors controlling mode of cellular separation after division in capsulated forms, transforming principles were obtained from the strain in question in many instances. Recovery of filamentous non-capsulated $(f i l+S-$ ) and of non-filamentous capsulated $(f i l-S+$ ) forms following growth of a non-filamentous non-capsulated (fil- $S-$ ) form in the presence of T.P. obtained from a putatively filamentous capsulated $(f i l+S+)$ variant demonstrated conclusively the genetic structure of the latter. Similarly, isolation of non-filamentous non-capsulated ( $f i l-S-$ ) and of filamentous capsulated ( $f i l+S+$ ) variants following the growth of a filamentous non-capsulated $(f i l+S-)$ strain in the presence of T.P. from a putatively non-filamentous capsulated (fil $-S+$ ) strain established the genetic constitution of the latter.

In experiments concerned with the possible exchange of desoxyribose nucleic acids controlling the production of type-specific capsular polysaccharide, a modified technique of transformation (9) was employed. The capsulated strain to be tested was inoculated in the manner described above into tubes containing T.P. from a pneumococcus of heterologous capsular type and into tubes without T.P. which served as controls. As the medium contained agglutinins against non-capsulated pneumococcal variants but lacked agglutinins directed against type-specific capsular antigens, an environment inimical to the selection of noncapsulated variants was maintained. After incubation of the cultures for 24 hours at $37^{\circ} \mathrm{C}$., 10 gamma of crystalline desoxyribonuclease (Worthington Biochemical Sales Co., lot D333) in $0.2 \mathrm{cc}$. of charcoal-absorbed broth were introduced into each tube and the cultures were reincubated for 1 hour. Appropriate controls showed this time to be adequate for destruction of the biological activity of the T.P. Thereafter, $1.6 \mathrm{cc}$. of each culture was transferred to a tube containing $4 \mathrm{cc}$. of neopeptone broth to which 10 per cent anticapsular rabbit serum directed against the inoculated strain had been added. The cultures were incubated overnight and the several morphologic variants were recovered and identified by the same cultural, serologic, and biologic procedures described above.

3. Strains of Pnermococcus.-The strains employed were those described in the preceding paper. One additional strain of pneumococcus capsular type I, strain IF ( $f l-S I$ ), and its morphologic variants were also used. This strain, isolated in Denmark in 1943, and designated I $519 / 4341^{\circ} \times 300$ by Dr. Erna M $\phi$ rch-Lund (10), was kindly sent by her from the State Serum Institute, Copenhagen.

\section{EXPERIMENTAL}

In Table I are shown the pneumococcal morphologic variants which might be expected to result from the interaction of the several variants with the transforming principles of heterologous forms. As indicated in the experimental data which follow, it has been possible to isolate eighteen of the twenty predicted recombinants. Because combination of the filamentous form with various capsular types led to the more ready identification of the several morphologic variants on blood agar plates, several strains of pneumococcus were used to facilitate the experiments.

Transformation of the Filamentous Non-Capsulated (fil $+S-$ ) Variant.When strain IID39S $(f l+S-$ ) was grown in the presence of the T.P. of strain IID39S ( $f l-S-$ ), non-filamentous non-capsulated $(f i-S-$ ) variants were recovered. This transformation was described originally by Taylor (6). 
Growth of strain IF (fll+S-) in the presence of the T.P. of strain ID ( fil $+S \mathrm{I}$ ) resulted in the isolation of the filamentous capsulated strain IF $(f i l+S I t r)$.

Transformation of the filamentous non-capsulated strain IID39S ( fil $+S-$ ) with the T.P. of the non-filamentous capsulated pneumococcus VIIIB ( $f l$ $S$ VIII) yielded three new morphologic variants: a filamentous capsulated strain $(f i l+S V I I I t r)$, a non-filamentous non-capsulated strain $(f i l-\operatorname{tr} S-)$, and a non-filamentous capsulated strain ( $f l-t r S V I I I t r)$. The presence of the two genetic factors controlling non-filamentous growth and production of type VIII capsular polysaccharide in the doubly transformed, non-filamentous

TABLE I

Morphologic Variants of Pneumococcus Arising from the Interaction of Cells of One Morphologic Variant with the Transforming Principles of an Heterologous Variant

\begin{tabular}{|c|c|c|c|c|}
\hline \multirow{2}{*}{ T.P. } & \multicolumn{4}{|c|}{ Strain } \\
\hline & $f i l+S-$ & fil $-S-$ & $f i l+S Y$ & fil $-S Y$ \\
\hline$f l+s-$ & - & $f l+t r S-$ & - & $f i l+t r S Y$ \\
\hline$f l-S-$ & fll-tr $S-$ & - & fil-tr $S Y$ & - \\
\hline$f i l+S X$ & $f i l+S X t r$ & $\begin{array}{l}f i l-S X t r \\
\text { fll+tr } S- \\
(\text { filt } \operatorname{tr} S X t r)\end{array}$ & $f i l+S X t r$ & $\begin{array}{l}f i l-S X i r \\
f l+t+S Y \\
(f i l+\operatorname{tr} S X t r)\end{array}$ \\
\hline$f l-S X$ & $\begin{array}{l}f i l+S X t r \\
f i l-t r S- \\
\text { fil-tr } S X t r\end{array}$ & $f l l-S X t r$ & $\begin{array}{l}f l l+S X t r \\
f i l-t r S Y \\
f i l-t r S X t r\end{array}$ & $f l-S X t r$ \\
\hline
\end{tabular}

$f l l+$, filamentous; $f l l-$, non-filamentous; $S X, S Y$, capsulated organisms of different capsular type; $\mathrm{S}-$, non-capsulated.

Symbols in parentheses indicate predicted forms not isolated.

capsulated ( $f l-S+$ ) variant was demonstrated through the effect of T.P. prepared from it upon a filamentous non-capsulated strain $(f i l+S-)$, resulting in the recovery of non-filamentous non-capsulated $(f i l-S-)$ and filamentous capsulated $(f i l+S+)$ forms.

Transformation of the Non-Filamentous Non-Capsulated (fil-S-) Variant. -Growth of the non-filamentous non-capsulated variant, IID39S (fil-S-), in a transforming system containing the T.P. of strain IID39S ( fll $+S-$ ) yielded filamentous non-capsulated ( $f l+S-$ ) forms as demonstrated first by Taylor (5)

Transformation of strain IID39S $(f l l-S-$ ) in the presence of the T.P. of the filamentous capsulated strain ID $(f i l+S I)$, resulted in the recovery of two new morphologic variants: a filamentous non-capsulated strain $(f i l+i r$ $S-$ ) and a non-filamentous capsulated strain ( $f l-S \mathrm{I} t r)$. The doubly transformed strain, (fil+tr $S \mathrm{I} t r)$, was not recovered in this experiment. This fail- 
ure may have resulted from the lack of selective factors favoring the growth of filamentous forms. Unlike non-filamentous variants which grow diffusely in liquid media, filamentous forms manifest varying degrees of autoagglutinability and are therefore not favored selectively as are the former in cultures containing both types of cells. As the probability of occurrence of a double transformation is in all likelihood the product of the individual probabilities of the two distinct transformations involved in the reaction, a selective factor may be necessary to facilitate its detection. The absence of such a selective factor from the experimental system may account for the failure to recognize doubly transformed cells.

Interaction of the non-filamentous non-capsulated strain, IID39S ( $f l-$ $S-$ ), with the T.P. of the non-filamentous capsulated strain, IIIA66 ( $f l-$ $S I I I)$, resulted in the recovery of non-filamentous capsulated type III pneumococci. This reaction is the classical example of pneumococcal capsular type transformation, discovered originally by Griffith (4) and elucidated by Avery, MacLeod, and McCarty (11).

Transformation of the Filamentous Capsulated (fil $+S+$ ) Variant.-In these experiments, the filamentous capsulated strain, IID39S (fil+ SVIIItr), was used because of its morphologic properties detectable in the gross. When grown in the presence of T.P. from the strain IF ( $f l-S-$ ), transformation of strain IID39S (fil + SVIIItr) to the non-filamentous capsulated variant ( $f i l-i r S V I I I t r)$ occurred. Although growth in liquid media favors selection of non-filamentous mutants arising spontaneously from filamentous forms, detection of such mutants requires usually several transfers in liquid media. In the controls employed in this experiment, no non-filamentous forms were observed. Two other aspects of this experiment merit comment. First, transformation took place in the absence of antibody capable of agglutinating the strain undergoing transformation and second a character of a capsulated pneumococcus appeared capable of transformation without evident loss of capsulation.

The possible transformation of pneumococcal capsular type through the mediation of desoxyribose nucleic acid exchange reactions has been discussed in a previous preliminary report (9). Although definitive proof of exchange reactions involving this pneumococcal character is lacking, study of reactions between capsulated strains of pneumococcus is not without interest. Experiment has shown that when the filamentous capsulated strain IID39S filt SVIIItr) is transformed in the presence of the T.P. of a filamentous capsulated strain of heterologous type, e.g. strain ID $(f i l+S \mathrm{I})$, according to the modified technique described previously, filamentous capsulated type I cells may be recovered from the system. Whether or not this reaction is mediated through the development of filamentous non-capsulated forms which acquire the new capsular type cannot be ascertained with certainty.

Employing the modified technique referred to above, transformation of the 
filamentous capsulated variant, IID39S ( $f l+S$ VIII $r$ ), with the T.P. of the non-filamentous capsulated strain, IIIA66 (fil-SIII), of heterologous capsular type, has resulted in the recovery of three new pneumococcal strains: a non-filamentous capsulated type VIII strain (fil-tr SVIII $t r$ ), a filamentous capsulated type III strain ( $f i l+S I I I t r)$, both of which represent single transformations and a doubly transformed strain ( $f l-t r S I I I t r)$. In this experiment, recovery of the doubly transformed variant is favored selectively by its properties of diffuse growth and by the absence of agglutinating antibody.

Transformation of the Non-Filamentous Capsulated (fil- $S+$ ) Variant.In this series of experiments, the non-filamentous capsulated variants, IID39S ( $f i l-S I I)$ and IID39S ( $f l-S V I I I t r)$, were used. Growth of the latter strain in the presence of T.P. from the filamentous non-capsulated strain, IID39S $($ fil $+S-$ ), resulted in the isolation of a filamentous capsulated pneumococcus $(f i l+S+)$. The reaction was carried out in an environment lacking agglutinins for the diffusely growing strain undergoing transformation demonstrating again that transformation of diffusely growing pneumococci may be recognized if selective factors favor detection of the transformed cells. In this instance, transformation of a character of a capsulated pneumococcus occurred under conditions unfavorable to the selection of spontaneous mutations to the filamentous variant recovered from the transforming system. The result supports the view that capsulated pneumococci may participate in transformation reactions without loss of capsulation.

With the use of the modified technique of transformation, the non-filamentous capsulated strain, IID39S (fil-SII), was transformed with T.P. from the filamentous capsulated strain, IID39S $(f i l+S I I I r)$. Although the doubly transformed variant was not recovered, a filamentous capsulated type II strain, (fil + tr $S \mathrm{II}$ ) and a non-filamentous capsulated type III strain ( $f i l-$ $S \mathrm{III} t r)$ were isolated. Lack of factors favoring selection of the doubly transformed variant, (fil+tr SIIItr) militated probably against its detection.

The possible exchange of capsular type has been demonstrated also through the interaction of the cells of a non-filamentous capsulated variant with the T.P. of a second non-filamentous capsulated variant of heterologous capsular type in the modified transformation reaction. Strain IID39S (fil-SIIItr) was recovered following growth of strain IID39S (fil-SVIIItr) in the presence of T.P. from strain IIIA66 ( $f l-S I I I)$. This reaction has been described previously in detail (9).

\section{DISCUSSION}

Studies with selective cultural techniques have indicated previously that morphologic variation in pneumococcus is determined chiefly by the mode of cellular separation after division and by the ability or inability of the cell to produce capsular polysaccharide. In addition, it has been shown that the genetic factors governing these two characters of pneumococcus are almost 
certainly desoxyribose nucleic acids which are inherited independently of one another. If these observations are correct, it should be possible to predict the results of transformation reactions involving competent pneumococci of one morphologic variant and the transforming principles obtained from cells of a different morphologic variant in a fashion analogous to the prediction of recombinations of independent characters in sexual forms. Experimental study of the predicted reactions has resulted in the isolation of all but two of the morphologic variants expected to result from such interactions. The undetected variants were those least likely to be found on theoretical grounds, representing double transformations arising in the absence of any selective factor to favor their discovery following interaction of non-filamentous noncapsulated $(f i-S-)$ and non-filamentous capsulated $(f i l-S X)$ strains with the transforming principles of a filamentous capsulated $(f i l+S Y$ ) strain of heterologous capsular type.

These data reveal also that transformation of diffusely growing pneumococci may take place in the absence of agglutinating antibody and that such transformations may include alteration of mode of cellular separation after division and acquisition of new capsular type as well as the acquisition of resistance to penicillin by diffusely growing cells demonstrated previously by Hotchkiss (12). The results show also that capsulated pneumococci may participate in transformation reactions involving characters other than the capsule of the pneumococcal cell.

\section{SUMMARY}

The interaction of each of the four morphologic variants of pneumococcus with the transforming principles of the heterologous morphologic variants of the same species has been studied and has resulted in the isolation of eighteen of the twenty variants predicted to arise through such interactions. The analogy of the findings to the recombination of independently heritable characters in sexually reproducing forms has been noted.

\section{BIBLIOGRAPHY}

1. Austrian, R., J. Exp. Med., 1953, 98, 21.

2. Griffith, F., Great Britain Rep. Pub. Health and Med. Subj., Ministry of Heallh, No. $18,1923,1$.

3. Dawson, M. H., J. Path. and Bact., 1934, 39, 323.

4. Griffith, F., J. Hyg., 1928, 27, 113.

5. Taylor, H. E., Compt. rend. Acad. sc., 1949, 228, 1258.

6. Taylor, H. E., J. Exp. Med., 1949, 89, 399.

7. Austrian, R., and MacLeod, C. M., J. Exp. Med., 1949, 89, 451.

8. MacLeod, C. M., and Krauss, M. R., J. Exp. Med., 1947, 86, 439.

9. Austrian, R., Bull. Johns Hopkins Hosp., 1952, 90, 170.

10. Mørch-Lund, E., Acta path. et microbiol. scand., 1949, 26, 709.

11. Avery, O. T., MacLeod, C. M., and McCarty, M., J. Exp. Med., 1944, 79, 137.

12. Hotchkiss, R. D., Cold Spring Harbor Symp. Quant. Biol., 1951, 16, 457. 